\title{
Aspergilosis esfenoidal, una sospecha poco habitual
}

\author{
Sphenoid aspergillosis, an unusual suspect
}

Miguel Alberto Rodríguez P1' Juan R. Benito Navarro, Eulalia Porras $\mathrm{A}^{1}$.

\section{RESUMEN}

La enfermedad aislada del seno esfenoidal constituye sólo el 2\%-3\% de toda la patología sinusal y en la mayoría de los pacientes es de tipo inflamatorio. Así mismo, es poco frecuente la invasión fúngica de dicho seno esfenoidal en un individuo sano e inmunocompetente, siendo extremadamente rara en niños.

Nos planteamos recordar esta patología haciendo presentación de nuestra última experiencia y obtener conclusiones para mantener siempre un espectro amplio en cuanto a los diagnósticos diferenciales a tener en cuenta.

日 presente caso clínico expone la experiencia vivida en nuestro centro con una patología poco frecuente como es la aspergilosis esfenoidal aislada, con los antecedentes personales, características clínicas, métodos diagnósticos, pronóstico y tratamientos óptimos.

Presentamos el caso de un varón de 73 años con historia de cefaleas intensas de larga evolución inicialmente sensibles a tratamiento analgésico, de localización retroocular bilateral, y que en los últimos 2 meses se hacen resistentes al tratamiento y comienzan a irradiarse a región naso-frontal derecha con mayor intensidad y con sonofobia, fotofobia, cortejo neurovegetativo, pérdida de peso, dolor cervical frecuente, intenso prurito ocular y ocasionalmente visión borrosa. Sin rinorrea, fiebre, ni ningún otro síntoma propio de un cuadro infeccioso.

Los síntomas iniciales de la enfermedad del seno esfenoidal son poco precisos, de instauración insidiosa y crónica, y difíciles de caracterizar. Hasta el 70\%, se manifiestan por alteraciones visuales que generalmente, indican complicaciones inmediatas. Está asociadaa peor pronóstico que en otras localizaciones por la posible extensión intracraneal precoz

Así, podemos considerar que la aspergilosis fulminante de senos paranasales en general, y del seno esfenoidal particularmente, representa una importante causa de morbi/mortalidad en pacientes cuyo estado inmunitario es deficiente, pero actualmente el patrón de comportamiento de los hongos está cambiando. Así se muestra en este caso en el que el paciente mantenía un sistema inmunitario competente.

Palabras claves: Aspergilosis, seno esfenoidal, cefalea, pérdida de visión, meningitis.

\section{ABSTRACT}

Isolated sphenoid sinus disease represents only 2-3\% of the total sinus pathology, and when it is present, it usually has an inflammatory origin. It seldom affects an immunocompetent person, and it is very rare in children.

Our goal is to draw attention to this pathology introducing our recent experience, and to draw conclusions in order to always bear in mind a wide spectrum of possible differential diagnoses.

Unidad Clínica de ORL. Hospital Universitario Puerta del Mar. Cádiz. España. 
We present the clinical experience at our hospital with a rare pathology such as isolated sphenoidal aspergillosis, reviewing the patient's personal background, clinical features, diagnosis techniques, prognosis and optimal therapy.

Our patient is a 73-year-old male with long-term intense headache located in the frontal and periocular regions. Initially, common analgesics were strong enough to relieve the pain, but eventually it became more and more intense, with no response to medication. In addition, he presented cervical pain, photophobia, sonophobia, weight loss, ocular tingling and blurred vision. There was no rhinorrea, fever, or any other infectious symptom.

Initial sphenoid sinus pathology symptoms are not very precise, with a latent and chronic set up, and they are also hard to characterize. Up to $70 \%$ of patients show visual disturbances, which generally derive in immediate complications.

Prognosis is worse than in other location because of early intracranial dissemination.

Thus, sphenoid aspergillosis can be considered as being a potentially lethal

disease in immunodeficient patients, but nowadays, fungal behavior is changing. This was the case here, as the patient remained immunocompetent.

Key words: Aspergillosis, sphenoid sinus, headache, visual loss, meningitis.

\section{INTRODUCCIÓN}

La enfermedad aislada del seno esfenoidal constituye sólo el 2\%-3\% de toda la patología sinusal, con un origen en la mayoría de los casos de tipo inflamatorio'.

Así mismo, es poco frecuente la invasión fúngica de dicho seno esfenoidal en un individuo sano e inmunocompetente, siendo extremadamente rara en niños. Además, está asociada a un peor pronóstico que en otras localizaciones por la posible extensión intracraneal precoz y la consiguiente meningoencefalitis.

La afectación por aspergilosis invasiva de una persona inmunocompetente es rara, aportándose tan sólo 17 casos en un período de 30 años en una de las últimas revisiones publicadas ${ }^{2,3}$.

Los síntomas iniciales de la enfermedad del seno esfenoidal son poco precisos y difíciles de caracterizar. A pesar de las variadas y críticas estructuras anatómicas, como la arteria carótida interna, la duramadre o los pares craneales II, III, IV, V y VI, los síntomas debidos a la afectación de estas estructuras son menos frecuentes que otros menos específicos, entre los que destaca la cefalea ${ }^{4}$.

Una vez extendida la infección desde el seno, el control es difícil con antifúngicos convencionales y tratamiento quirúrgico, con una elevada tasa de mortalidad $^{5}$. Así, podemos considerar que la aspergilosis esfenoidal es una enfermedad potencialmente letal ${ }^{6}$.

Posiblemente la infección nasosinusal fulminante por mucormicosis en personas inmunodeprimidas sea una entidad bien conocida, mientras que la destrucción masiva de los senos paranasales por aspergilosis es menos frecuente y menos sospechada ${ }^{7}$.

Quizás el uso creciente de corticosteroides, drogas citotóxicas y otros tratamientos médicos inmunosupresores, así como patologías como diabetes mellitus, cirrosis hepática, malnutrición, deshidratación y procesos tumorales, o simplemente la evolución en las técnicas diagnósticas y el conocimiento de la enfermedad, pueden ser los motivos que explican el aumento de su incidencia8.

Por las características anatómicas del seno esfenoidal, con una menor circulación de flujo aéreo en comparación con el seno maxilar, la afectación aislada de aquél es poco frecuente.

Los síntomas de esta patología son variados: cefalea, dolor retroocular, diplopia, exoftalmos y ceguera. El más frecuente y síntoma princeps es la cefalea frontal y retroorbitaria, 0 de tipo vago y poco definida, que se agrava con ciertos movimientos como tos, caminar, esfuerzos, pudiendo interferir seriamente en el sueño con una respuesta pobre a los analgésicos.

Son muy habituales, hasta en $70 \%$, las alteraciones visuales que implican a los pares craneales 
II, III, IV y VI, y que generalmente indican complicaciones inmediatas.

La afectación del nervio trigémino se produce en el $10 \%-30 \%$.

Por otro lado, la afectación nasal no es frecuente, lo cual nos indica que la afectación esfenoidal no tiene porqué ser consecuencia de una rinosinusitis previa ${ }^{6,9,10}$.

Una de las principales características son la instauración insidiosa y crónica, con retraso y posibles errores en el diagnóstico, lo que conlleva complicaciones por extensión secundaria intracraneal u orbitaria. De esta forma, las complicaciones suponen una menor tasa de recuperación completa.

Para un manejo efectivo de la aspergilosis esfenoidal se requiere un diagnóstico precoz mediante pruebas de imagen (TC, RMN, endoscopía), determinación histopatológica, cirugía y antibióticos, y en algunas ocasiones quimioterapia o corticosteroides ${ }^{11}$.

La disponibilidad cada vez más habitual de TC y RMN como métodos de diagnóstico por imágenes, así como de la endoscopía nasal, ha permitido un aumento en la frecuencia de diagnóstico de enfermedad aislada del seno esfenoidal.

El diagnóstico definitivo se realiza mediante estudios histológicos de la mucosa sinusal y/o de la masa en el interior del seno.

La infección fúngica de los senos paranasales por Aspergillus puede manifestarse como:

- Forma aguda fulminante: En pacientes inmunodeprimidos con alta tasa de morbi/ mortalidad por su extensión y diseminación hematógena.

- Forma indolente en inmunocompetentes. Generalmente originada por Aspergillus y con asiento en el seno esfenoidal, provocando inflamación crónica, limitada y circunscrita, sin extenderse a tejidos vecinos.

- Forma micetoma: Sin erosión ósea pero con esclerosis del hueso que la contiene, y ligera respuesta inflamatoria.

- Forma alérgica: Cursa como una infección crónica en pacientes adultos jóvenes inmunocompetentes y átopicos con afectación nasal por obstrucción e incluso poliposis ${ }^{12,13}$.

\section{CASO CLíNICO}

Varón de 73 años cuyos antecedentes médicos de interés son: ser ex-fumador, ex-bebedor, haber presentado una reacción adversa a carbamacepina en el pasado, fiebre de Malta hace 25 años, espondiloartrosis y ulcus gástrico; y que ha sido intervenido de apendicectomía y herniorrafia inguinal. Sin realizar tratamiento actualmente.

El paciente es remitido desde un hospital comarcal con historia de cefaleas intensas de 2 años de evolución que inicialmente cedían con tratamiento analgésico, de localización retroocular bilateral, pero que en los últimos 2 meses se han hecho resistentes al tratamiento y se han comenzado a irradiar a región naso-frontal derecha con mayor intensidad y acompañadas de sonofobia, fotofobia, cortejo neurovegetativo, así como sensible pérdida de peso, dolor cervical frecuente, intenso prurito ocular y ocasionalmente visión borrosa. No se acompaña de rinorrea, fiebre, ni ningún otro síntoma que haga sospechar un cuadro de características infecciosas.

La exploración ORL y neurológica realizada tanto en su hospital de origen como en nuestro centro son rigurosamente normales.

En cuanto a las pruebas complementarias realizadas:

- Hemograma/bioquímica/coagulación: Dentro de la normalidad.

- Serología vírica, brucela, espiroquetas, sin hallazgos patológicos.

- Pruebas tiroídeas: Dentro de la normalidad.

- TC craneal: Sin hallazgos patológicos.

- TC senos paranasales: "Ocupación y abombamiento de seno esfenoidal izquierdo con discontinuidad de techo óseo y pared posterior de dicho seno".

- RMN: "Lesión localizada en seno esfenoidal, de aspecto redondeado, bien delimitada, hipointensa en T2 y hiperintensa en T1. Hay realce periférico de la lesión siendo compatible con mucocele esfenoidal sin captaciones patológicas intracraneales" (Figura 1).

Ante este diagnóstico de mucocele esfenoidal, el centro de origen decide derivar al paciente al Servicio de Neurocirugía para valoración de actuación quirúrgica. Durante su estancia se repite TC 


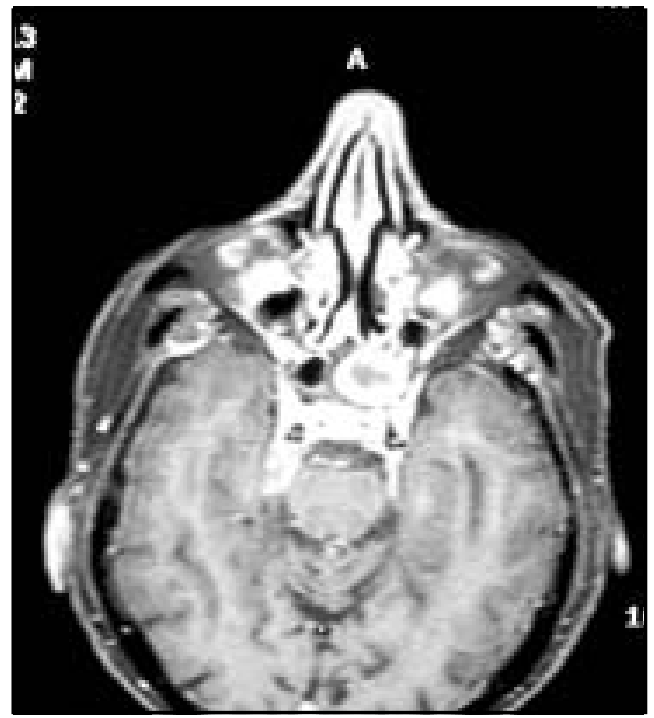

Figura 1. Aspecto de la lesión en RMN.

de senos paranasales y se observa "aumento de densidad del hueso esfenoidal afectando a ambos senos esfenoidales, de aspecto algodonoso y compatible en primer lugar con displasia fibrosa esfenoidal. Así mismo, ocupación y abombamiento de seno izquierdo con pared lateral y superior, esta última prácticamente desaparecida por su delgadez. En definitiva, hallazgos clínicos y radiológicos compatibles con displasia fibrosa afectando al esfenoides, con mucocele de seno esfenoidal izquierdo" (Figuras 2 y 3 ).
Dada la persistencia de la cefalea, que se mantiene con localización naso-órbito-frontal bilateral, pero con predominio derecho, y de potente intensidad que impide el descanso, se inicia terapia analgésica por parte de la Clínica del Dolor de nuestro hospital.

En el curso de los días presenta episodios de visión borrosa unilateral derecha y ptosis parcial, por lo que se consulta con los Servicios de Oftalmología y Neurología. Ambos emiten informes dentro de la normalidad una vez realizadas sus

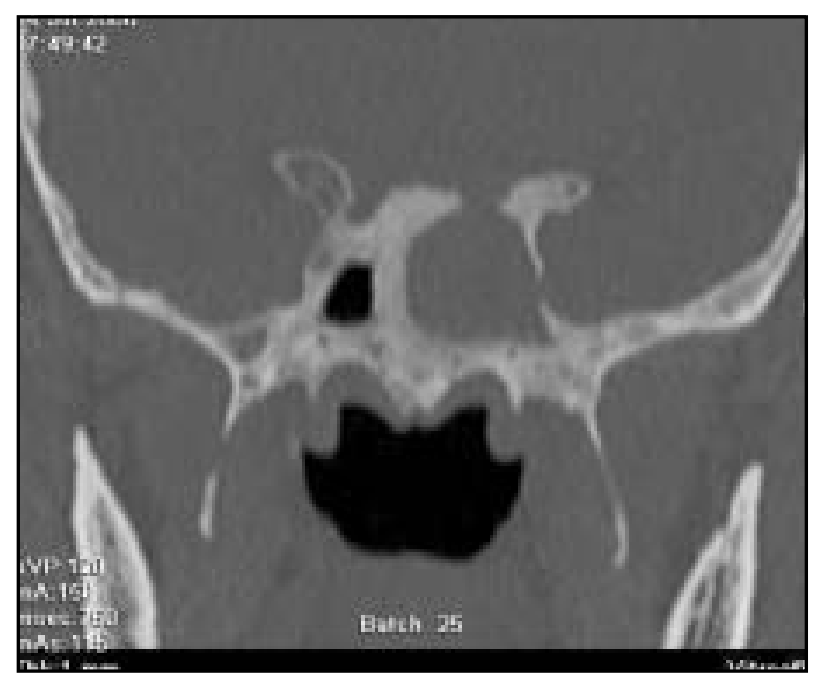

Figura 2. Aspecto de la lesión en TAC. 


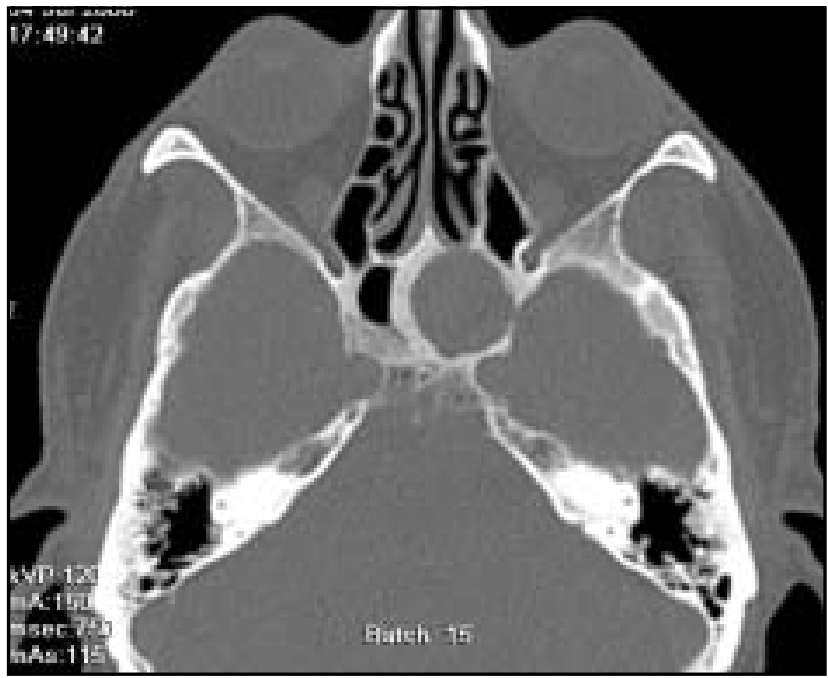

Figura 3. Aspecto de la lesión en TAC.

exploraciones por lo que se mantiene el diagnóstico de cefalea crónica secundaria a mucocele esfenoidal.

El Servicio de Neurocirugía decide derivar el paciente al hospital de referencia neuroquirúrgica para intervención. Allí se realiza biopsia de la masa ocupante de espacio en seno esfenoidal, y es enviada a estudio por el Servicio de Anatomía Patológica. El paciente es dado de alta con leve mejoría sintomática, quedando pendiente de informe anatomopatológico de la pieza.

Posteriormente el paciente precisa reingreso en nuestro centro por nueva sintomatología dolorosa intensa y picos febriles, que tras realización de hemocultivos, no dan resultados clarificadores. Durante este nuevo ingreso se produce ceguera bilateral, confirmada mediante campimetría, y ptosis derecha completa, informando el Servicio de Oftalmología "amaurosis bilateral por fallo en la aferencia del par craneal II de ambos ojos, de carácter brusco; posiblemente de causa vascular 0 compresiva en ambos nervios ópticos. Carácter irreversible dada la pérdida de visión".

Por su parte, neurología informa de "atrofia óptica de ojo izquierdo. Pupilas isocóricas no reactivas a la luz. Amaurosis bilateral".

El informe histológico de la pieza es el siguiente: "múltiples acúmulos constituidos por conglomerados de hifas y esporas con las características de
Aspergillus. Se identifican también fragmentos de mucosa con infiltrados inflamatorios agudos y subagudos con pequeños focos de polimorfonucleares alrededor de los vasos, cuya luz también está rellena por dichos hongos. Así mismo, también se aprecian acúmulos de hongos en muestras de fragmentos óseos. Ausencia de elementos de carácter tumoral en los límites de la muestra".

Una vez conocido dicho informe anatomopatológico, se contacta con el Servicio de Medicina Interna, que inicia tratamiento antifúngico con voriconazol de manera inmediata.

Pasadas 24 horas tras el comienzo de la terapia antibiótica, el paciente fallece por paro cardiorespiratorio.

\section{DISCUSIÓN/CONCLUSIONES}

La aspergilosis fulminante de los senos paranasales en general, y del seno esfenoidal en particular, representa una importante causa de morbi/mortalidad en pacientes cuyo estado inmunitario es deficiente, pero actualmente el patrón de comportamiento de los hongos está cambiando, como así lo demuestra este caso, en el que el paciente no presenta déficit inmunitario.

El Aspergillus es un hongo disperso por todo el mundo, con especial intensidad en otoño e invier- 
no, y al cual la mayoría de las personas es inmune. Está presente tanto en el ambiente como en el suelo y la materia orgánica, y su principal mecanismo de entrada al organismo es mediante inhalación, a partir de la cual inicia un crecimiento saprofítico, pudiendo convertirse en patógeno bajo ciertas condiciones anaeróbicas. Así, personas con puesto de trabajo expuesto al ambiente, como jardineros, granjeros, pueden estar predispuestos a una colonización por dicho agente.

El establecimiento de los síntomas en un paciente por lo general sano, es habitualmente indolente pero incesante y progresivo.

Los síntomas de la afectación de este seno se refieren a las estructuras próximas antes que al propio seno. El síntoma predominante es la cefalea, con incidencias que varían entre $33 \%-81 \%$, según las series estudiadas. Algunos autores, incluso, establecen que la cefalea retroocular es típica de la enfermedad del seno esfenoidal, al igual que en nuestro paciente 4 .

La localización característica de esta cefalea está en relación con la inervación del seno esfenoidal, que deriva de fibras del $V_{1}$ par craneal y aferencias vía ganglio esfenopalatino.

El siguiente síntoma en frecuencia es la afectación de la visión, pudiendo limitarse a ligeras pérdidas de campo visual y leves pérdidas de la agudeza, o bien llegar a producirse una grave ceguera e incluso amaurosis, asociada a ptosis e imposibilidad de movilizar el globo ocular. En este caso los síntomas son secundarios a la afectación del nervio óptico y del motor ocular externo, que además es uno de los más precozmente lesionados. En general, la afectación de este órgano sensorial se cifra en $24 \%-50 \%$.

La afectación de las fosas nasales es muy infrecuente (en algunas series del $4 \%)^{10}$.

El diagnóstico de la lesión del seno esfenoidal se basa en la utilización de las técnicas de imagen (TC y RMN), así como la endoscopía nasal, obteniendo la confirmación diagnóstica siempre con la histopatología, que además nos informa de la extensión del proceso ${ }^{1}$.

Las imágenes de TC y RMN nos permiten conocer información detallada que incluye consideraciones anatómicas, extensión de la lesión y destrucción sobre tejidos vecinos.

La utilización de la endoscopía nasal nos permite identificar lesiones sutiles en meato medio, surco olfatorio y ostium esfenoidal, y la toma de biopsias de secreciones liberadas por la lesión con actuaciones terapéuticas precoces y con mínima morbilidad.

Cada vez podemos diagnosticar este tipo de patología con mayor prontitud y tratarlas de una manera más segura sin afectación de las estructuras adyacentes que podría comprometer el pronósticos.

El diagnóstico diferencial que debemos plantearnos debe incluir las siguientes entidades: mucormicosis, granuloma de línea media, patología vascular, sinusitis fúngica alérgica 0 lesiones tumorales de las fosas y senos paranasales ${ }^{4}$.

En cuanto al tratamiento, no hay consenso, ya que algunos autores preconizan un abordaje endoscópico transnasal con el que se realice de manera efectiva la limpieza sinusal, mientras que otros prefieren abordaje externo, especialmente cuando las órbitas están implicadas.

Existen controversias en cuanto al uso de anfotericina $B$ tras la cirugía de desbridamiento en la aspergilosis. Algunos autores prefieren itraconazol, y otros ambos en conjunto. Actualmente en uso creciente el voriconazol.

En cualquier caso, existen series que estudian los resultados del tratamiento con itraconazol, que afirman que en el momento en que la infección se convierte en meningoencefalitis, las dosis habituales de este antifúngico $(8 \mathrm{mg} / \mathrm{kg} / \mathrm{d})$ no son efectivas y deben doblarse para obtener resultados aceptables ${ }^{15,16}$.

Otros estudios afirman que cualquier tipo de terapia antibiótica es inefectiva ante esta patología una vez que consigue extenderse, de manera que si se convierte en intracraneal, la mortalidad alcanza el $100 \%$ sin tratamiento ${ }^{17,18}$.

Las tasas de recuperación completa aportadas en pacientes con enfermedad limitada al seno esfenoidal y tratados con antibioterapia intensiva y drenaje quirúrgico alcanzan el $30 \%{ }^{18}$.

Esta patología se plantea como un verdadero reto tanto para el cirujano ORL como para el oftalmólogo y el neurocirujano, los cuales deben mantener siempre en mente esta posibilidad ante una cefalea crónica a veces difusa, pérdida de visión, imposibilidad de movimiento ocular junto a ptosis, y sabiendo que los síntomas nasales pueden estar absolutamente ausentes? 


\section{BIBLIOGRAFÍA}

1. Castelnuovo P, Pagella F, Semino L, de Bernardi, F, DELU G. Endoscopic treatment of the isolated sphenoid sinus lesions. Eur Arch Aorhinolaryngol 2005; 262: 142-7.

2. Sivak-Callcott J, Livesley N, Nugent R, Rasmussen S. Localized invasive sino-orbital aspergillosis: characteristic features. Br J Ophtalm 2004; 88 : 681-7.

3. Deligny C, Le Thi Huong D, Grivois JP, Clero D, BodaghI B, Piette JC. Optic neuropathy revealing invasive aspergillosis of ethmoid and sphenoid sinuses. La revue de médecin interne2005; 26 : 425-6.

4. Martin T, Smith T, Smith M, Loehrl T. Evaluation and surgical management of isolated sphenoid sinus disease. Arch Otolaryngol. Head Neck Surg 2002; 128: 1413-9.

5. Takahiko Y, Shibano K, Soeda T, Hoshi A, Matsuura Y, Sugiura Y, Endo K, Yamamoto T. Intracraneal invasive aspergillosis originating in the sphenoid sinus: a successful treatment with high-dose itraconazole in three cases. Tohoku J Exp Med 2004; 20: 133-9.

6. Ruoppi P, Seppä J, Pukkila M, Nuutinen J. Isolated sphenoid sinus diseases: report of 39 cases. Arch. Otolaryngol. Head Neck Surg 2000; 126 (6): 777-81.

7. Chopra H, Dua K, Malhotra V, Gupta R, Puri $H$. Invasive fungal sinusitis of isolated sphenoid sinus in immunocompetent subjects. Mycoses 2006; 49: 30-6.

8. Harrill W, Stewart M, Lee A, Cernoch P. Chronic rhinocerebral mucormycosis. Laryngoscope 1996; 106: 1292-7.

9. Kanoh N, Xu R, Wang Z, Dal C, Chi F, Kutler D, Tian X. Isolated sphenoid sinus disease: an analysis of 122 cases. The Annals of Otology, Rhinology \& Laryngology 2002; Apr; 111: 4.

10. Lawson W, Reino AJ. Isolated sphenoid sinus disease: an analysis of 132 cases. Laryngoscope 1997; 107: 1590-5.

11. Wenzel S, Sagowski C, Kehri, Metternich FU. Course and therapy of an invasive aspergilloma of the skull base in a nonimmunocompromised patient. HNO2004; Aug. 52 (8): 724-8.

12. Botturi A, Salmaggi A, Pollo B, Lamperti e, ERbetta A, Bolardo A. Meningitis following relapsing painful ophtalmoplegia in aspergillus sphenoidal sinusitis: a case report. Neurol Sci 2006; 27: 284-7.

13. Manfred P, Honegger J, Daschner F, Feuerhake $F$, Zentner J. Fungal granuloma of the sphenoid sinus and clivus in a patient presenting with cranial nerve III paresis: case report and review of the literature. Neurosurgery 2003; 52(4): 955-9.

14. Proetz AW. Operation of the sphenoid sinus. Trans Am Acad Ophtalmol Otolaryngol 1949; 53: 538-45.

15. Perrin C, Boutard P, Reman 0. Fatal cerebral aspergillosis in acute megakarioblastic leukemia. Arch Fr Pediatr 1989; 46: 45-8.

16. Sánchez C, Mauri E, Dalmau D, Quintana S, Aparicio A, Garau J. Treatment of cerebral aspergillosis with itraconazole: do high doses improve the prognosis? Cin Infect Dis 1995; 21: 1485-7.

17. DuPONT B. Itraconazole therapy in aspergillosis: study in 49 patients. J Am Acad Dermatol 1990; 23: $607-14$.

18. Iplikcioglu AC, Bek S, Bikmaz K, Ceylan D, GöKDUMAn CA. Aspergillus pituitary abscess. Acta Neurochir 2004; 146: 521-4.

Dirección: Miguel Alberto Rodríguez Pérez.

Avda. Amílcar Barca 47, 60 E. 11009 Cádiz. Teléfono: 677836605.

otomiguel@gmail.com 